\title{
Impressum, Vol. 2, No. 4, 1979
}

\section{Herausgeber}

Prof. Dr. S. Eckhardt, Staatliches Onkologisches Institut, Budapest

Prof. Dr. R. Gross, Medizinische Universitätsklinik, Köln

Prof. Dr. J. H. Holzner, Institut für pathologische Anatomie,

Universität Wien

Prof. Dr. K. Munk, Deutsches Krebsforschungszentrum, Institut für

Virusforschung, Heidelberg

Prof. Dr. G. P. Murphy, Roswell Park Memorial Institute,

Buffalo (N.Y.)

Prof. Dr. A. Stacher, Hanusch-Krankenhaus, Wien

Prof. Dr. Dr. St. Tanneberger, Zentralinstitut für Krebsforschung,

Akademie der Wissenschaften, Berlin/DDR

Prof. Dr. W. Wilmanns, Medizinische Klinik III, Klinikum Groß-

hadern, Universität München

Prof. Dr. Dr. H. Wrba, Institut für Krebsforschung, Universität Wien

Die Zeitschrift erscheint zweimonatlich; pro Jahr erscheint 1 Band zu je 6 Heften.

Bezugspreis für Jahrgang 2, 1979 DM 104,-/ÖS 728,-/SFr. 104,-. 1 Einzelheft kostet DM 22,-/ÖS 154,-/SFr. 22,-, einschließlich MwSt., zuzüglich Postgebühren.

Der Abonnementspreis ist im voraus zahlbar. Die Lieferung der Zeitschrift läuft weiter, wenn sie nicht spätestens 4 Wochen vor Abschluß eines Bandes abbestellt wird.

Abonnementsbestellungen können bei jeder Buchhandlung, bei der Post oder direkt beim Verlag aufgegeben werden: Bundesrepublik Deutschland/österreich: S. Karger GmbH, Post-fach 2, D8034 Germering/München, Tel: (089) 844021, Telex: 524865 D, Postscheckkonto: München 400 80-807 Schweiz: S. Karger AG, Arnold-Böcklin-Straße 25, CH-4011 Basel, Tel.: (061) 3908 80, Telex $62652 \mathrm{CH}$

Anzeigen

S. Karger Verlag für Medizin und Naturwissenschaften GmbH, Postfach 2, 8034 Germering, Telefon (0 89) 844021.

Wissenschaftlicher Beirat:

H. Denck, Wien; V. Diehl, Hannover; F. Douwes, Göttingen; P. Drings, Heidelberg; H. Ehrhart, München; J. Fischer, Mainz; W. M. Gallmeier, Nürnberg; H. Gerhartz, Berlin; E. H. Graul, Marburg/Lahn; E. Hauptmann, Zagreb; H. Heimpel, Ulm; K. P. Hell-riegel, Köln; Ch. Herfarth, Ulm; R. Hünig, Basel; W. Hunstein, Heidelberg; H. O. Klein, Köln; H. Löffler, Lahn Gießen; U. Mohr, Hannover; F.-L. Müller, Bonn; G. Nagel, Göttingen; A. Pfleiderer, Freiburg; Mildred Scheel, Bonn; E. Scherer, Essen; S. Seeber, Essen; H. J. Senn, St. Gallen; W. Vahlensieck, Bonn; H. D. Waller, Tubingen; M. Wannenmacher, Freiburg; K. Wilms, Tubingen; K. zum Winkel, Heidelberg

Produktions- und Anzeigenleitung: Werner Zuckschwerdt 
Satz und Druck: Buch- und Offsetdruckerei Walter Biering KG, 8000 München 40

Für den Inhalt außerhalb des redaktionellen Teiles (insbesondere Anzeigen,

Industrieinformationen, Pressezitate und Kongreßinfor-mationen) übernehmen Herausgeber und Verlag keine Gewähr.

Alle Rechte, insbesondere das Recht der Vervielfältigung und Mikro-kopie sowie der

Übersetzung, vorbehalten. Nachdruck, auch auszugs-weise. nur mit Genehmigung des Verlages.

(C) Copyright 1979 by S. Karger Verlag für Medizin und Naturwissenschaften GmbH, Postfach 2,

D-8034 Germering

Offizielles Organ der Deutschen Gesellschaft für Hämatologie und Onkologie und der

österreichischen Gesellschaft für Hämatologie und Onkologie

Hinweise für Autoren

Bitte senden Sie Ihr Manuskript direkt an den Verlag S. Karger.

Es werden nur Originalarbeiten in deutscher Sprache veröffentlicht.

Das Manuskript soil in druckreifem Zustand in dreifacher Ausferti-

gung eingereicht werden. Die Entscheidung über die Aufnahme erfolgt

aufgrund mehrerer Gutachten. Eine Kopie des Manuskripts soil grund-

sätzlich beim Autor verbleiben.

Die typographische Gestaltung und redaktionelle Bearbeitung ist dem

Verlag vorbehalten.

Der Verlag erwirbt alle Rechte an Sonder- und Abdrucken in anderen

Publikationen. Autorkorrekturen über 10\% der Satzkosten hat der

Autor zu tragen. Dem Autor stehen 40 Gratissonderdrucke seiner

Arbeit zu.

Markennamen müssen, wenn sie geschützt sind, mit einem hochge-stellten ${ }^{\circledR}$ bezeichnet werden.

Die Verantwortung für die korrekte Befolgung dieser Vorschrift liegt ausschließlich beim Autor. Abkürzungen sollen möglichst sparsam verwendet werden. Bei spe-ziellen Abkürzungen sollte der Autor jeweils beim ersten Auftreten das Wort ausschreiben und die später verwendete Abkürzung in Klam-mern folgen lassen: z.B. Adenosin-monophosphat (AMP).

Institutsangabe in der Sprache der Arbeit.

Zusammenfassungen sollen 20 Schreibmaschinenzeilen nicht über-schreiten. Eine Fassung in englischer Sprache und der englische Titel der Arbeit müssen mitgeliefert werden, auch wenn die Arbeit in deutscher Sprache abgefaßt ist. In die Zusammenfassung gehören keine Literaturzitate, Tabellen, Abbildungen und Abbildungshinweise sowie allgemein gehaltene Aussagen.

Schlüsselwörter sollen ebenfalls in deutscher und englischer Sprache abgefaßt werden. Zu einer Arbeit gehören drei bis fünf Key Words.

Tabellen, Abbildungen und Literaturzitate müssen im Text erwähnt werden.

Abbildungsvorlagen dürfen nicht aufgeklebt, gelocht oder mit Büro-klammern angeheftet eingereicht werden und sollen auf der Rückseíte den Namen des Autors, den Titel des Beitrags sowie die Bildnummer tragen.

Adresse des Autors ist am Schluß der Arbeit vollständig anzugeben.

Literaturverzeichnis. Es soil nur die im Text zitierten Publikationen, diese dafür lückenlos, enthalten. Die Literatur ist entweder alpha-betisch zu ordnen oder entsprechend der Reihenfolge im Text zu numerieren. 